\title{
Verde epidota: pintando el pasado en Cerro de Oro, valle de Cañete, Perú. Un análisis Químico y mineralógico del PIGMENTO CERÁMICO VERDE AMARILLENTO
}

\author{
Adrián González-Gómez de Agüero ${ }^{2}$, Francesca Fernandini Parodi ${ }^{\mathrm{b}}$, \\ Luis Ortega-San-Martin ${ }^{c}$ y Patricia Gonzales Gild
}

\begin{abstract}
Resumen
El siguiente estudio propone identificar uno de los pigmentos más característicos del valle de Cañete durante el periodo Horizonte Medio, especificamente, el color verde amarillento observado en el repertorio cerámico de Cerro de Oro. Definir el origen y utilización de este pigmento facilita el entendimiento de la obtención de materia prima y cadena operativa en la elaboración de una de las cerámicas que definen a este desarrollo regional. Para este análisis se han utilizado como muestras, cerámicas con pigmento verdoso y muestras de rocas con restos de un mineral verdoso, halladas ambas en el sitio arqueológico de Cerro de Oro. Para definir la composición quimica y mineralógica, se han llevado a cabo análisis de FRX, Raman y DRX. A partir de estos análisis, se ha podido determinar que el mineral encontrado es epidota y su comportamiento a altas temperaturas parece indicar que este mineral podría estar relacionado con el pigmento encontrado en las cerámicas del sitio.
\end{abstract}

Palabras clave: Cerro de Oro, Cañete, Perú, Andes, Horizonte Medio, pigmentos, epidota, FRX, Raman, DRX.

\section{Abstract \\ GREEN EPIDOTE: PAINTING THE PAST IN CERRO DE ORO, CAÑETE VALLEY, PERU. A CHEMICAL AND MINERALOGICAL ANALYSIS OF THE GREEN-YELLOWISH CERAMIC PIGMENT}

The following study proposes to analyze one of the most characteristic pigments of the Cañete Valley during the Middle Horizon period, specifically, the green-yellowish color in the Cerro de Oro ceramic repertoire. Defining the origin and use of this pigment allows for a better understanding of the access to raw materials and the operational chain in the manufacture of one of the ceramics that define this regional development. Ceramic fragments with the greenish pigment and epidote samples, both found at the Cerro de Oro archaeological site, have been used as samples for this study. To define the chemical and mineralogical composition of the pigment, XRF, Raman and XRD analyses were carried out. These analyses have allowed us to identify epidote as the colored mineral, and its behavior at high temperatures suggests that this mineral could be related to the pigment found in the ceramics of the site.

Keywords: Cerro de Oro, Cañete, Peru, Andes, Middle Horizon, pigments, epidote, XRF, Raman, XRD.

a https://orcid.org/0000-0002-5296-005X. Pontificia Universidad Católica del Perú. adrian.gonzalez@pucp.pe

b https://orcid.org/0000-0001-6530-8637. Pontificia Universidad Católica del Perú. ffernandini@pucp.edu.pe

c https://orcid.org/0000-0001-8779-0794. Sección Química, Departamento de Ciencias, Pontificia Universidad Católica del Perú. lortegas@pucp.pe

d https://orcid.org/0000-0003-0136-5813. Sección Química, Departamento de Ciencias, Pontificia Universidad Católica del Perú.pgonzales@pucp.pe 


\section{Introducción}

La cerámica andina prehispánica es conocida en todo el mundo por su iconografía y su impresionante combinación de colores. De hecho, el color ha sido durante décadas una de las principales herramientas para clasificar las cerámicas según su cronología, procedencia y cultura. Detrás de la selección de estos esquemas de colores se encuentra una compleja cadena de operaciones que incluye aspectos sociales y tecnológicos.

Actualmente, las nuevas técnicas en el estudio de la fabricación de cerámica nos ayudan a comprender cómo era la explotación del medio ambiente y la interacción entre las diferentes comunidades. Dependiendo de las técnicas analíticas usadas, es posible obtener información acerca del origen y la recolección de la materia prima, así como de cómo se trabaja esta, lo cual permite comprender mejor las capacidades tecnológicas desarrolladas en un lugar específico (Velde y Druc 1999). Es por eso que el estudio de los pigmentos es una herramienta útil para profundizar en el conocimiento sobre las identidades de las comunidades en el pasado y su tecnología.

$\mathrm{El}$ presente estudio continúa con una robusta y creciente línea de investigación en la arqueología latinoamericana vinculada al estudio de materiales y su tecnología a partir de la arqueometría. Un reflejo de este auge de la arqueometría latinoamericana es la publicación de Chapoulie et al. (2018), que por primera vez sistematiza en un texto en español el conocimiento de las técnicas arqueométricas, incluyendo casos de estudio tanto de América como de Europa. En cuanto a los estudios de pigmentos, se ha generado una gran producción bibliográfica que aborda su uso sobre distintos materiales. En la línea de este crecimiento y sistematización de estos estudios destaca la reciente colección Pigments, dyes, and colors in Latin american archaeometric investigations publicada en Heritage Science (Sepúlveda y Casanova 2020). En cuanto a la región que nos ocupa, este tipo de análisis ha adquirido gran relevancia en la literatura arqueológica peruana de las últimas décadas. Como apuntan Del-Solar-Velarde et al. (2020), el desarrollo de estos estudios en el área de los andes centrales, se puede ver reflejado en la creciente muestra de encuentros científicos vinculados a la arqueometría. Cabe destacar para el caso de Perú, la inclusión por primera vez en 2014 de una mesa entorno al estudio arqueométrico en el Congreso Nacional de Arqueología organizado por el Ministerio de Cultura. Además, y con un mayor énfasis en el análisis de pigmentos, cabe mencionar el simposio internacional Avances en el análisis de cerámica y pigmentos, organizado en junio de 2018 por la Pontificia Universidad Católica del Perú, que culminó con la publicación de dos compendios de algunas de las investigaciones en esta misma revista: Boletín de Arqueología PUCP 26 y 27 (2019): Avances en el análisis de cerámica y pigmentos en arqueología (Partes 1 y 2).

En este estudio proponemos analizar el origen y uso de uno de los pigmentos más utilizados en el repertorio cerámico del sitio arqueológico Cerro de Oro: el verde. Las diferentes investigaciones realizadas en el sitio arqueológico Cerro de Oro han reportado que una particular tonalidad del verde descrita como verde amarillento o verde pistacho es utilizado tanto como engobe, como en el pintado de diseños geométricos y figurativos. Es decir, a menudo forma parte del soporte que acompaña la iconografía del sitio y a su vez, se integra en los motivos iconográficos. También ha sido hallado como pigmento principal de algunas cerámicas escultóricas. De alguna manera, este color ha pasado a ser reconocido por los arqueólogos que trabajan la costa centro-sur, como un color asociado a la identidad de los habitantes de Cerro de Oro. Asimismo, algunos estudios de reconocimiento y prospección a lo largo de Cerro de Oro han identificado, adherido al monzogranito que compone la roca madre (Salazar y Landa 1993; Fernandini 2015: 90), un mineral de coloración verdosa, que podría haber sido utilizado como materia prima para la elaboración del pigmento verde amarillento. En este sentido, proponemos que identificar la cadena operativa detrás de la obtención, procesamiento y uso de dicho pigmento nos acercará a entender los procesos técnicos y sociales detrás del uso de este color tan significativo en el sitio.

Para llevar a cabo esta investigación nos hemos centrado en tres objetivos principales: 
Figura 1. Ubicación geográfica del sitio Cerro de Oro (elaboración: Kelly Gómez).

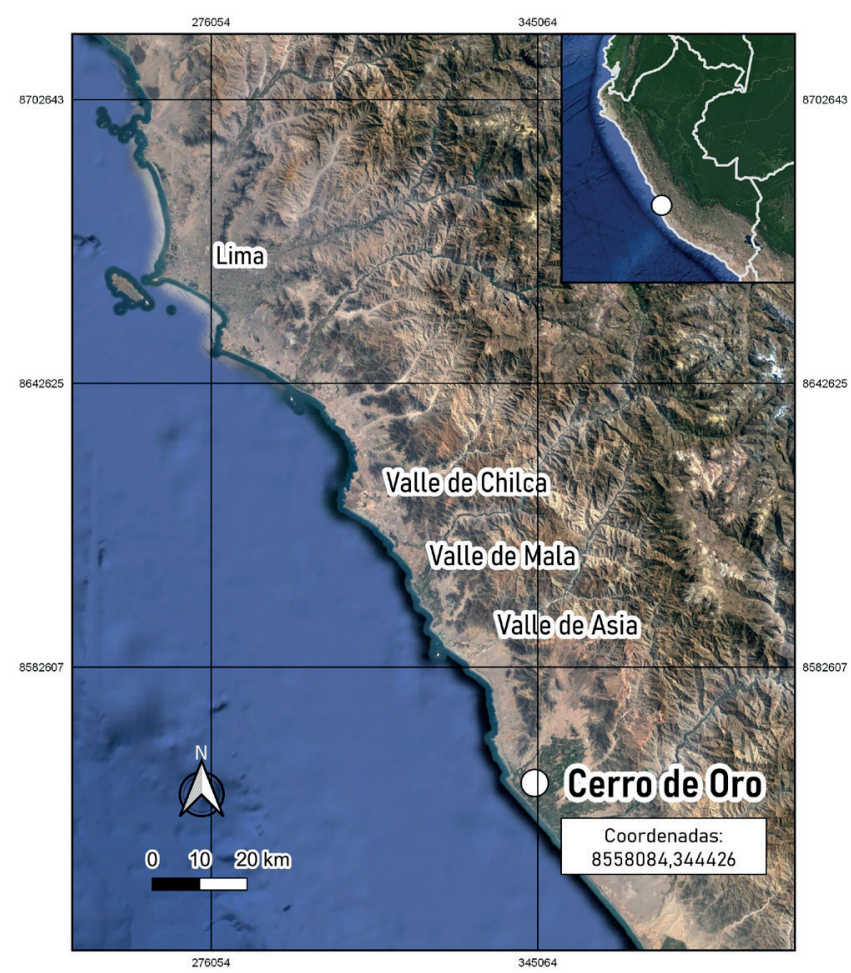

- Analizar la composición química y mineralógica del mineral verdoso encontrado en el sitio.

- Analizar la composición química y mineralógica de los pigmentos verdosos de la cerámica de Cerro de Oro y relacionarla con el mineral encontrado en el sitio.

- Estudiar cómo se transforma el mineral a altas temperaturas para poder establecer una relación con el pigmento utilizado en las cerámicas.

De esta manera pretendemos conocer si existe una correlación entre el mineral y los pigmentos verdosos hallados en el sitio de Cerro de Oro.

\section{Cerro de Oro}

Esta investigación se ha llevado a cabo en el sitio arqueológico Cerro de Oro (Fig. 1), un asentamiento urbano ubicado en el valle bajo de Cańete, siendo la principal área urbana de la zona entre 550-950 d.C. (Kroeber 1937; Menzel 1964; Stumer 1971; Ruales 2000; Fernandini 2015, 2018a,b; 2020). A lo largo de aproximadamente 300-400 años de ocupación, Cerro de Oro se desarrolla como un asentamiento urbano que congregó a una población de más de 8000 personas (Fernandini 2018b). Esta población construyó y habitó un sitio muy organizado, donde predominaron los barrios residenciales, las áreas de reunión y las zonas de almacenamiento, todos caracterizados por tener altas paredes que limitaban la visibilidad y el tránsito. Igualmente, recientes investigaciones centradas en la dieta (González-Gómez de Agüero 2019) y la forma de vida (Fernandini 2020) en estos barrios residenciales indican la presencia de posibles subdivisiones sociales dentro del sitio, donde cada barrio estaría representando a comunidades con distintas tradiciones, prácticas culinarias, entre otras (Fernandini 2018b). 
Cerro de Oro fue un desarrollo local que ejerció una fuerte influencia en los valles vecinos de Asia, Mala y Chilca. Igualmente, el estudio de su cerámica, textiles y otros soportes materiales, revela que los pobladores de Cerro de Oro interactuaron activamente con sus vecinos de Lima (norte) y Nasca (sur). Esta interacción se refleja en la incorporación de figuras y colores representativos de las sociedades Lima y Nasca, dentro de una base claramente local. En este sentido, la población de Cerro de Oro se desarrolla localmente, con sus propios estándares y marcadores culturales, participando activamente del dinamismo cultural que caracterizó este período, donde ideas y personas parecen haber interactuado continuamente.

\section{La cerámica}

La cerámica Cerro de Oro fue estudiada de manera preliminar por Alfred Kroeber (1937), y luego incluida en el estudio realizado por Dorothy Menzel para toda la cerámica del Horizonte Medio (600-1000 d.C.). Sobre la base del estudio de la colección cerámica excavada por Kroeber, Menzel introduce el término «estilo» Cerro de Oro (Menzel 1964) y lo describe como un estilo ecléctico que incluye influencias Nasca, Lima, Wari, además de una clara base local. Posteriormente, Fernandini (2015) y Rodríguez (2017) también realizan análisis cerámicos y proponen una secuencia estilística cronológica que incluye una primera etapa donde el estilo Cerro de Oro presenta características predominantemente locales (550-700 d.C.), con un uso exclusivo de decoraciones geométricas, y un segundo momento que incluye la introducción de representaciones iconográficas provenientes de Nasca y Lima, sobre una base local (700-850 d.C.). Fernandini destaca la presencia de algunas piezas Wari de origen foráneo (Fernandini 2020). A lo largo de los c. 300 años de ocupación, la cerámica Cerro de Oro presenta un repertorio bastante estandarizado, con formas y decoraciones recurrentes, y particularmente con una tonalidad de colores homogénea. Mientras que los diseńos van cambiando e incluso se amplía la paleta de colores, hay dos colores que persisten a lo largo de toda la ocupación: el morado oscuro y el verde amarillento (Fig. 2) (Menzel 1964; Stumer 1971; Ruales 2000; Fernandini 2015; Rodríguez 2017). Estos colores han pasado a representar el estilo Cerro de Oro tanto dentro como fuera del sitio, en los distintos valles vecinos.

Estudios centrados en el color morado oscuro han revelado que este pigmento tiene un importante componente de titanio (Fernandini 2015); sin embargo, se requiere mayores investigaciones para conocer la procedencia y manera de preparación de este pigmento. En cuanto al color verde amarillento, se propone que una de las fuentes de materia prima podría ser un mineral de tonalidad similar que se puede encontrar en los distintos afloramientos rocosos que se presentan a lo largo del sitio. Este mineral aparece adherido al monzogranito que compone la roca madre. En ciertas ocasiones, este mineral verdoso aparece como parte del registro arqueológico, asociado a espacios de quema.

Finalmente, y en cuanto al uso de pigmentos verdes, estos son poco comunes entre las sociedades prehispánicas peruanas, a pesar de que existen algunas como Paracas (Kriss 2018; Dulanto et al. 2019) o Nasca, donde se da con mayor frecuencia. Además, se ha hallado de forma excepcional en contextos Moche (Wright 2010). Generalmente estos pigmentos suelen estar asociados con minerales cuyo componente principal es el cobre.

\section{El contexto}

Este estudio se ha centrado en fragmentos de cerámica hallados durante la temporada de campo 2017 del Proyecto Arqueológico Cerro de Oro (en adelante, PACO) (Fernandini 2018a). El contexto excavado corresponde a un recinto residencial/doméstico que presentaba áreas de actividad claramente diferenciadas para cocinar, almacenar, descansar, desechar basura, producir cerámica, entre otros. En general el contexto parece haber sido un área de uso comunal donde se realizaban 


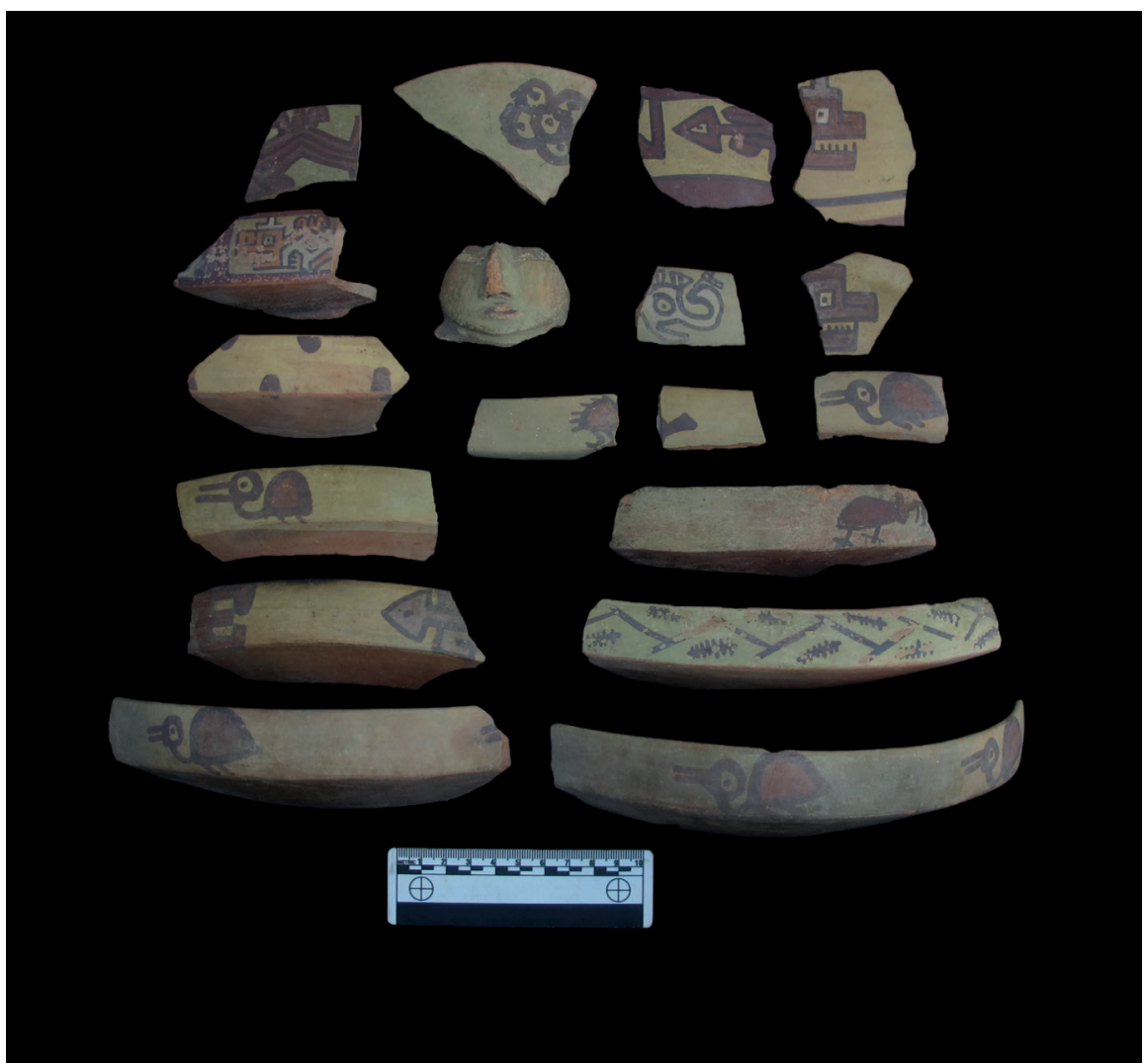

Figura 2. Fragmentos cerámicos con pigmento verdoso y decoración iconográfica hallados en la temporada 2017 del PACO. Muchos de ellos corresponden a la forma definida como cuenco Cerro de Oro (fuente: Fernandini 2015; Rodriguez 2017).

actividades relacionadas con la preparación de alimentos, así como con la producción cerámica. De particular interés para este estudio, fueron los hallazgos de un horno de cerámica y una gran zona de quema donde se registró la presencia de un sedimento verdoso mezclado con cenizas (Fernandini 2018a). Dentro de esta zona de quema se registró una serie de fragmentos cerámicos de distintas vasijas con pintura o engobe en verde amarillento. Adicionalmente, en áreas cercanas al espacio excavado se registraron fragmentos líticos color verde. Tanto la cerámica como los fragmentos líticos forman parte de la muestra base para el análisis del uso de pigmentos de este estudio.

\section{Metodología}

Este trabajo se ha centrado en estudiar el origen y uso del pigmento verde amarillento con el que se pintó las superficies de las vasijas cerámicas registradas en este contexto residencial, así como en el análisis de fragmentos de mineral verdoso hallado cercano a la zona de quema con sedimento verdoso. Para este propósito, se han realizado diferentes análisis utilizando técnicas de contraste para identificar si el pigmento mineral que hemos encontrado en el registro arqueológico podría ser el mismo que los habitantes de Cerro de Oro usaban para pintar sus recipientes. Primero, hemos analizado la composición química del mineral verdoso encontrado en el sitio. Posteriormente, analizamos la composición química del pigmento verdoso de la cerámica Cerro de Oro y lo relacionamos con el mineral que se encuentra en el sitio. Finalmente, hemos estudiado 
cómo se transforma el mineral a altas temperaturas para establecer una relación con el proceso de producción de cerámica.

El análisis de los pigmentos de tonalidad verde registrados en la cerámica Cerro de Oro requiere un enfoque que incluye diversas técnicas analíticas, así como dos tipos de muestra. Se aplicaron las técnicas de difracción de rayos X (DRX), espectrometría de fluorescencia de rayos X (FRX) y microscopía Raman en dos tipos de muestras: pigmentos verdes registrados en un grupo de fragmentos cerámicos provenientes de Cerro de Oro y un conjunto de fragmentos rocosos de coloración verdosa recogidos en el sitio.

Tabla 1. Relación de muestras analizadas en este estudio.

\begin{tabular}{lllll}
\hline $\begin{array}{c}\text { Número de } \\
\text { muestra }\end{array}$ & Tipo de muestra & Proveniencia de la muestra & Preparación & $\begin{array}{c}\text { Análisis practicados } \\
\text { sobre la muestra }\end{array}$ \\
\hline Muestra 01 & Cerámica & $\begin{array}{l}\text { Cuarto A, Capa A, Recinto } \\
\text { Quema, Locus A6 }\end{array}$ & Raspado & Raman \\
Muestra 02 & Fragmento rocoso & $\begin{array}{l}\text { Recolección superficial } \\
\text { En polvo }\end{array}$ & FRX, DRX, Raman \\
\hline
\end{tabular}

\section{Muestras cerámicas}

La muestra cerámica está compuesta por cinco fragmentos que fueron hallados en la estructura de quema descrita líneas arriba. Por su ubicación estratigráfica y por el tipo de iconografía, con influencias del estilo Nievería de la costa central, estos fragmentos pertenecen al segundo momento del estilo Cerro de Oro (700-850 d.C.). Todos los fragmentos seleccionados para esta muestra presentan en su superficie exterior, ya sea a manera de engobe o decoración, el color verde amarillento (Fig. 3). El color y los diseńos iconográficos de la superficie indican que estos fragmentos formaron parte de una misma pieza. Estos fragmentos fueron seleccionados debido tanto a su color como a la iconografía presente en su diseño, ya que esta combinación de factores nos habla de una etapa durante la cual la cerámica Cerro de Oro se redefinió incluyendo, tanto rasgos locales como foráneos.

Esta muestra, al estar centrada en fragmentos de una misma vasija, representa un estudio preliminar sobre el uso de materias primas en Cerro de Oro. Eventualmente, se plantea ampliar el repertorio de la muestra e incorporar análisis centrados en el procesamiento de preparación de los pigmentos, así como su aplicación sobre la cerámica.

\section{Fragmentos rocosos}

Se recogieron un total de ocho muestras de monzogranito con el mineral verdoso adherido (Fig. 4). Estas muestras fueron recogidas de la superficie en las zonas adyacentes al área de excavación de la temporada 2017. Actualmente no se ha hallado ninguna veta geológica del mineral en el sitio, sino que se encuentra asociado al monzogranito de forma dispersa. Es decir, no se ha podido observar en forma de cristales individuales crecidos sobre la roca, sino como parte del conglomerado de minerales de la misma. En consecuencia, no se pudo hacer una separación física del pigmento. Se tomaron los fragmentos de roca que contenían mayor coloración verde y se molieron para su análisis. Finalmente cabe destacar que únicamente fue necesario utilizar una pequeńa parte de una de las muestras para los análisis. 


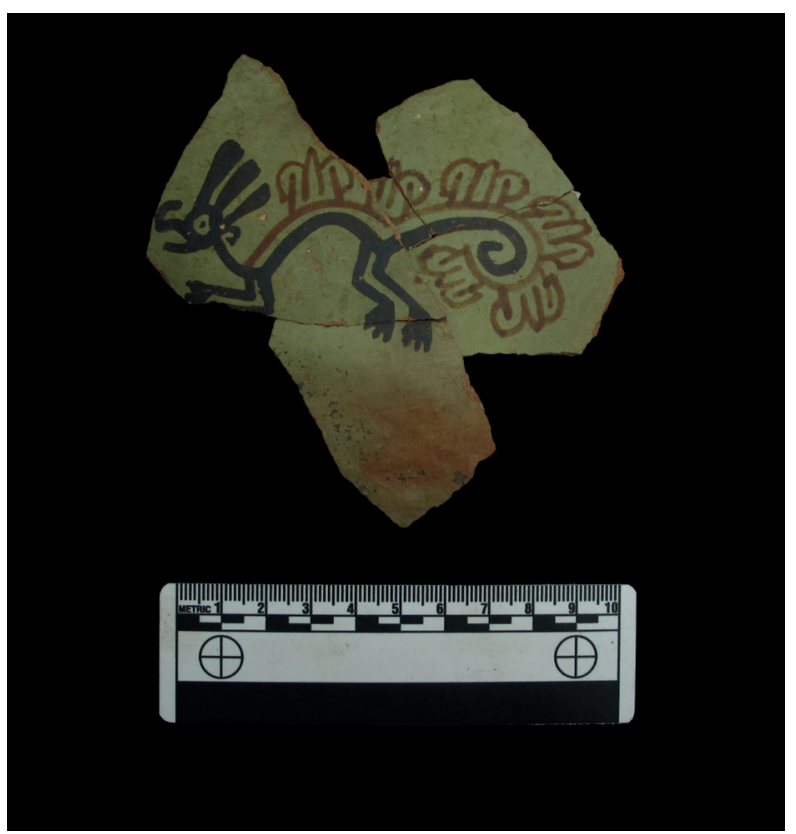

Figura 3. Fragmentos cerámicos con pigmento verdoso y decoración iconográfica hallados en la temporada 2017 del PACO. El motivo iconográfico corresponde al animal jorobado (humped-back animal). Sobre estos fragmentos se han realizado los distintos análisis.

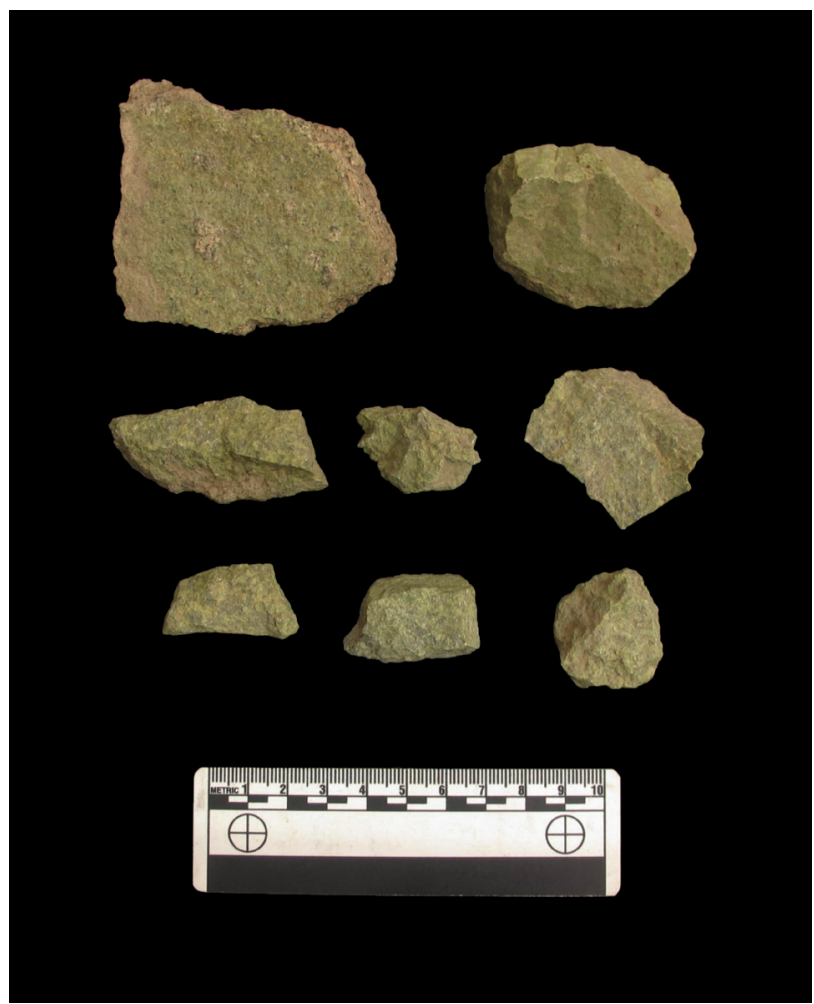

Figura 4. Fragmentos rocosos con el mineral verdoso adheridos, recogidos durante la temporada 2017 del PACO. 


\section{Métodos analíticos y técnicas empleadas}

\subsection{Preparación de las muestras}

En el caso de los fragmentos cerámicos, se realizaron análisis no invasivos, sin preparación de muestra, y también microdestructivos. Para estos últimos, se tomaron micromuestras raspando la superficie del fragmento cerámico con un bisturí y procurando no incluir la pasta en el raspado.

En el caso de los fragmentos rocosos recolectados, se seleccionaron las zonas de las rocas obtenidas con una mayor concentración del mineral verde. El material, fue molido manualmente con un mortero de ágata y sometido al análisis sin ningún proceso extra. Para los estudios de transformación a alta temperatura, el polvo fue prensado en pastillas que fueron sometidas a diferentes tratamientos térmicos. Se hicieron 6 pastillas de 10 milímetros de diámetro usando una prensa hidráulica. Para cada pastilla se usaron, aproximadamente, 0.3 gramos del polvo molido. Una de las pastillas no fue calentada, mientras que las otras se calentaron en un horno tipo mufla, controlando la temperatura y los tiempos de subida, exposición y descenso según el esquema mostrado en la figura 5. Las temperaturas máximas (Tmax) de calentamiento fueron $600{ }^{\circ} \mathrm{C}, 800{ }^{\circ} \mathrm{C}, 900{ }^{\circ} \mathrm{C}$, $950{ }^{\circ} \mathrm{C}$ y $1000{ }^{\circ} \mathrm{C}$. Las temperaturas fueron elegidas considerando un rango de temperaturas de cocción entre 800 y $1000^{\circ} \mathrm{C}$ para este tipo de cerámicas (Fernandini et al. 2020). La quema de las muestras se hizo en aire (atmósfera oxidante).

Las muestras fueron sometidas a los análisis descritos a continuación, para identificar su composición química y mineralogía. Los análisis fueron realizados en los laboratorios del Centro de Caracterización de Materiales (CAM) y en los laboratorios de la Sección Química de la Pontificia Universidad Católica del Perú.

\subsection{Espectrometría de fluorescencia de rayos $\mathrm{X}$ (FRX)}

Se utilizó un analizador portátil de espectrometría de fluorescencia de rayos X por energía dispersiva marca Bruker, modelo Tracer III - SD, equipado con un tubo de rodio. Las condiciones de análisis fueron las siguientes: voltaje de $40 \mathrm{kV}$, corriente anódica de $10,3 \mu \mathrm{A}$, tiempo de irradiación de 60 s, sin filtros y sin aplicación de vacío. La sensibilidad del detector permite analizar elementos desde el aluminio hasta el uranio. Los espectros fueron interpretados utilizando el software Spectra Artax versión 7.4.0.0. Ni se seleccionaron ni se descartaron picos durante el análisis. El análisis solo fue cualitativo.

\subsection{Difracción de rayos $\mathrm{X}(\mathrm{DRX})$}

Para identificar la composición mineralógica de los fragmentos rocosos hallados en el sitio arqueológico Cerro de Oro, se realizó un análisis de difracción de rayos X. Se usó un difractómetro de polvo modelo Discover DaVinci de la marca Bruker que dispone de un ánodo de cobre. El análisis se ha realizado usando un intervalo angular en $2 \theta$ de $7^{\circ}$ a $80^{\circ}$, con un paso de $0.020^{\circ}$ y con un tiempo de 0.20 segundos por paso. La identificación de minerales se hizo usando el software Diffrac.Suite EVA de Bruker y la base de datos Powder Diffraction File (PDF 2, -2001). La cuantificación de fases y la determinación del contenido cristalino se realizaron usando el software TOPAS 5.0 de Bruker.

\subsection{Microscopía Raman}

Los análisis fueron realizados en un espectrómetro Renishaw Invia (láser de 633 y $785 \mathrm{~nm}$ ) con el software WiRE 3.4. Se usó un barrido de 100 a $2200 \mathrm{~cm}^{-1}$ y un objetivo de 50X. Los parámetros para el análisis fueron variados de acuerdo a la muestra analizada. Se utilizaron tiempos de exposición entre 10 y 20 segundos por barrido por un total de 1 a 5 acumulaciones, y una potencia de láser de 0.05 a $5 \%$. 
Figura 5. Secuencia de calentamiento a alta temperatura seguido para todas las muestras. Debido al material refractario del horno el enfriamiento no sigue el programa a temperaturas cercanas a la temperatura ambiente.

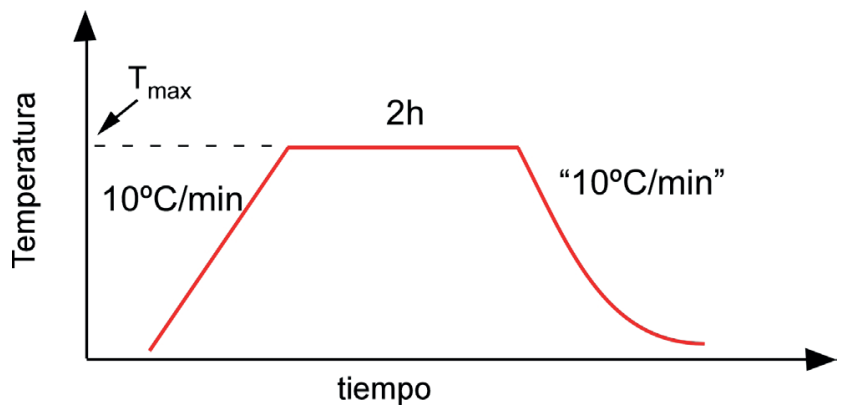

\section{Resultados y discusión}

A continuación, y con base en los análisis realizados, se evaluarán los objetivos principales del estudio presentados anteriormente. De esta forma se buscó identificar las composiciones químicas y mineralógicas del mineral encontrado en el sitio, así como del material cerámico, para comprobar si, finalmente, existía una relación entre ellas.

\subsection{Identificación del mineral verdoso}

\section{Espectrometría de fluorescencia de rayos X (FRX)}

En primer lugar, se realizó el análisis de fluorescencia de rayos $\mathrm{X}$ sobre el mineral verdoso. Las medidas se realizaron sobre la pastilla que no fue calentada. El espectro obtenido se muestra en la figura 6. Se identificaron elementos como el hierro, calcio silicio, titanio, azufre y seńales pequeñas pero inequívocas de aluminio y potasio. Debe recordarse que la intensidad observada no es necesariamente proporcional a la cantidad de cada elemento debido a los diferentes efectos de absorción cruzada en la matriz analizada. La ausencia de cobre descartó la posibilidad de que se tratara de minerales compuestos por este elemento, con lo que se descartaron todos los posibles pigmentos verdes basados en compuestos de cobre.

\section{Difracción de rayos $\mathrm{X}(\mathrm{DRX})$}

En la figura 7a se muestra el patrón de difracción de uno de los fragmentos de roca molidos y analizados por DRX. El análisis de los minerales presentes se realizó acotando los elementos presentes a los identificados por FRX y añadiendo todos aquellos que el equipo no permite identificar (elementos con número atómico menor a 13). Este análisis nos permitió identificar la presencia de dos minerales principales: cuarzo y epidota (aproximadamente el 97\% de la fracción cristalina) además de dos minerales en cantidades menores (calcita y clinocloro). La proporción de cada mineral se muestra en la figura $7 \mathrm{~b}$. Estos minerales son consistentes con el monzogranito de la zona. Dado que uno de los colores más comunes de la epidota es el verde, puede decirse que este es el mineral que le da el color verde a la roca recolectada (Klein y Hurlbut 1998: 342-343). Estos resultados nos invitan a pensar que la epidota podría ser la fuente del pigmento verde usado en las cerámicas.

\section{Microscopía Raman}

En la figura 8 se muestra el espectro Raman medido en uno de los granos verdes del polvo prensado del fragmento de roca molido que no fue calentado a alta temperatura. En la figura se incluye el espectro Raman de referencia de una epidota (Epidote R050191) de Pampa Blanca, provincia de Castrovirreyna, Huancavelica, Perú, tomada de la base de datos de la Universidad de Arizona (Lafuente et al. 2015). Como puede verse, ambos espectros tienen las mismas señales, lo que se encuentra en buen acuerdo con lo observado por difracción de rayos X en polvo. 


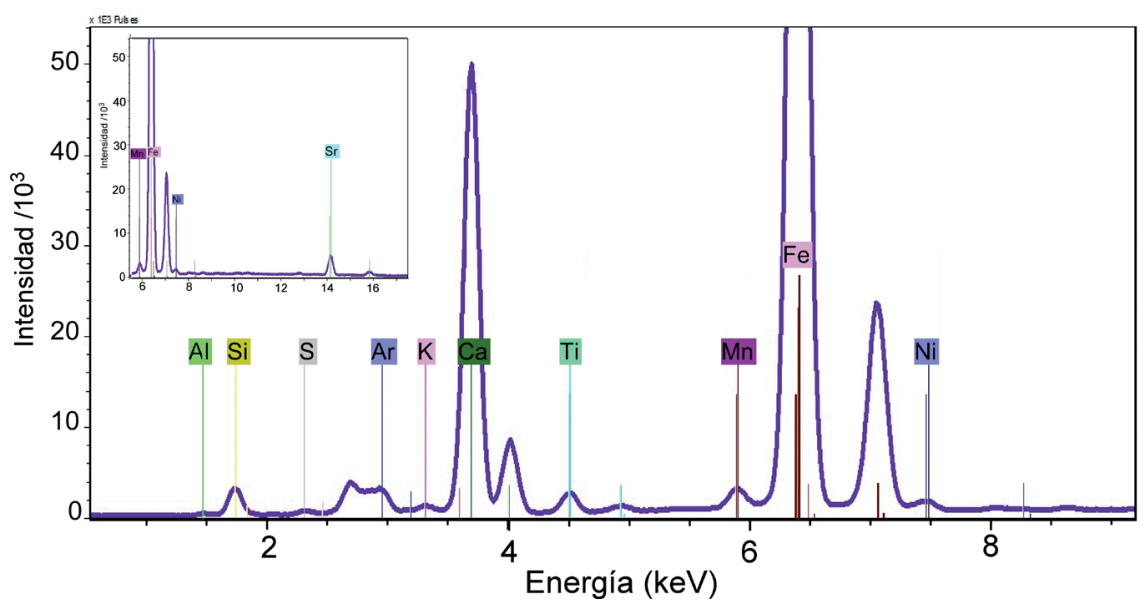

Figura 6. Espectro de fluorescencia de rayos $X$ por energía dispersiva del polvo molido de los fragmentos rocosos encontrados en Cerro de Oro. Se muestra la región entre 1 y $9 \mathrm{keV}$, que es donde se concentran las señales de los elementos de interés. Arriba a la izquierda se muestra la región de mayor energía que solo contiene la señal de un elemento extra.

Con base en los análisis antes descritos hemos podido concluir que el mineral verdoso hallado en el sitio de Cerro de Oro corresponde con la epidota. Esta epidota es un mineral sorosilicato de calcio, aluminio y hierro con un sistema cristalino monoclínico (Klein y Hurlbut 1998: 342-343), cuya fórmula idealizada es $\mathrm{Ca}_{2}\left(\mathrm{Fe}^{3+}, \mathrm{Al}\right) \mathrm{Al}_{2} \mathrm{O}\left(\mathrm{SiO}_{4}\right)\left(\mathrm{Si}_{2} \mathrm{O}_{7}\right)(\mathrm{OH})$. Una de las principales características de este mineral es su color verdoso que va del verde pistacho al verde amarillento, ambos muy comunes dentro del repertorio cerámico Cerro de Oro. Hoy en día, este mineral se vende como pigmento tanto en su forma original como molido (Kremer s/f). Además, se ha reportado la presencia de este mineral como pigmento en vasos queros de los períodos inca y colonial en Perú (Newman y Derrick 2002). Finalmente, cabe destacar que hemos observado este mineral en otros sitios arqueológicos cercanos como El Huarco (Cerro Azul), El Salitre (Mala) e Incahuasi (Lunahuaná), todos ubicados en la provincia de Cañete y con ocupaciones a partir del Período Intermedio Tardío. Sin embargo, este mineral no ha sido analizado y caracterizado aún.

\subsection{Identificación del pigmento verdoso de la cerámica}

\section{Microscopía Raman}

El análisis no invasivo de los fragmentos de cerámica por microscopía Raman no permitió obtener información sobre el pigmento verde presente en ellos. Por esta razón, se recurrió a la toma y análisis de micromuestras. El espectro Raman obtenido de las micromuestras del pigmento raspado de la cerámica es muy distinto al de la epidota (Fig. 9). La gran diferencia entre los espectros tiene dos posibles lecturas: o bien no se usó el pigmento encontrado o este fue aplicado a la cerámica previo a su proceso de cocción. En este último caso la epidota podría haberse alterado estructural y químicamente con lo que su espectro Raman característico ya no sería el mismo. Por esta razón, se planteó evaluar las posibles alteraciones químicas de la epidota a diferentes temperaturas de cocción. Cabe indicar que no se hicieron medidas de DRX de las micromuestras por ser una cantidad insuficiente para los análisis. 

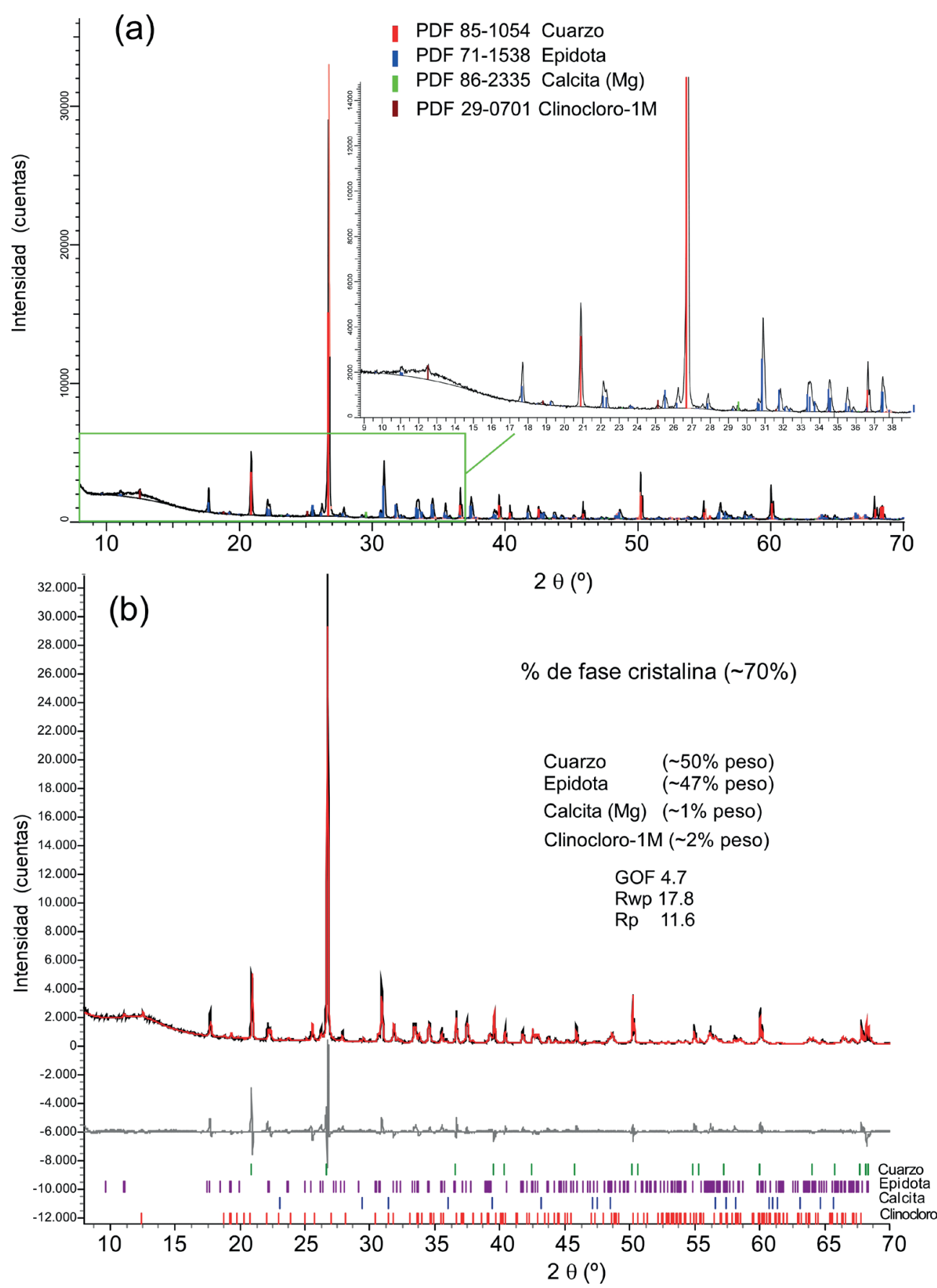

Figura 7. Difractogramas de la roca verde molida. (a) identificación de las fases observadas. Se muestra el código PDF de cada mineral en la base de datos Powder Diffraction File. La zona recuadrada en verde se ha ampliado arriba a la derecha para facilitar la identificación. (b) Afnamiento Rietveld de la muestra mostrada en (a). En negro está el patrón de difracción experimental y en rojo el calculado. En gris la diferencia. Las marcas verticales hacen referencia a los planos atómicos de cada mineral. Los resultados del afinamiento y los parámetros de ajuste se muestran en la figura. 


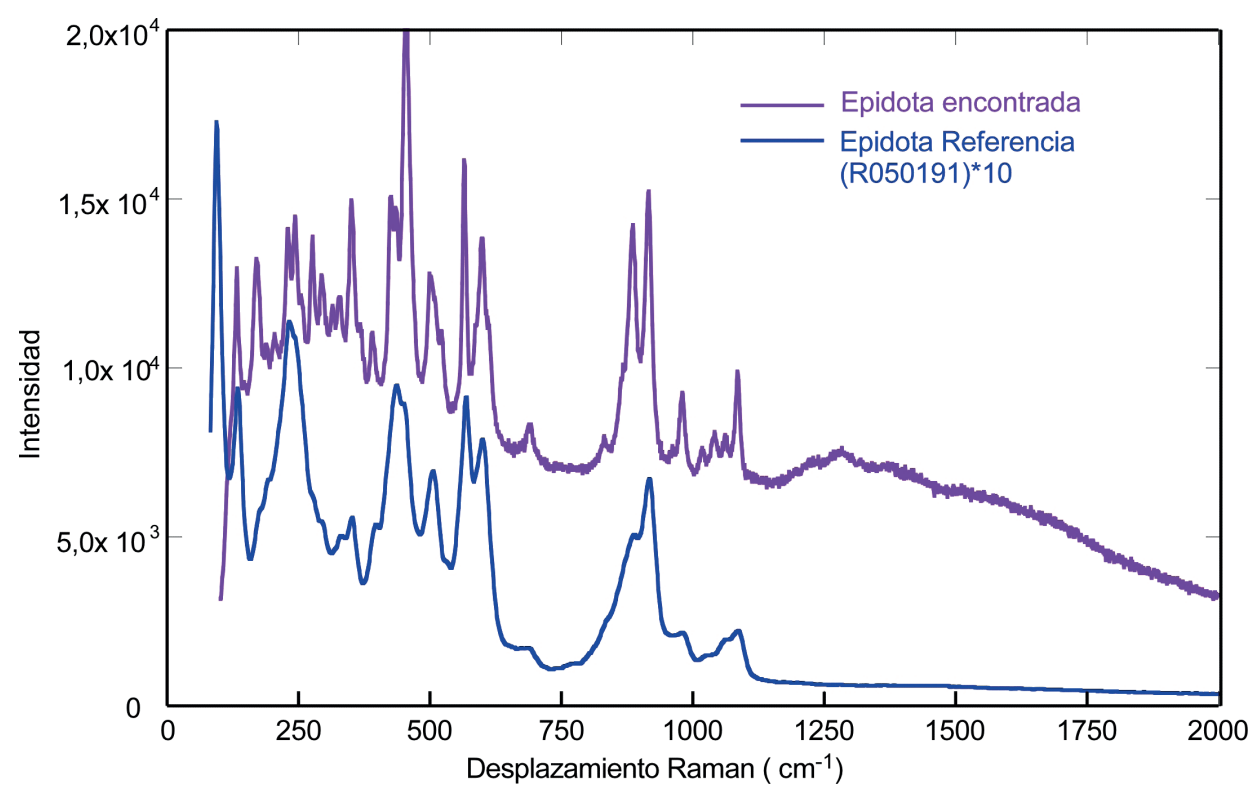

Figura 8. Espectros Raman del mineral verde encontrado en los fragmentos de roca recogidos en el sitio de Cerro de Oro y de un mineral epidota de referencia. Los datos de la epidota se tomaron usando un láser de $785 \mathrm{~nm}$. Para la referencia se usó $780 \mathrm{~nm}$.

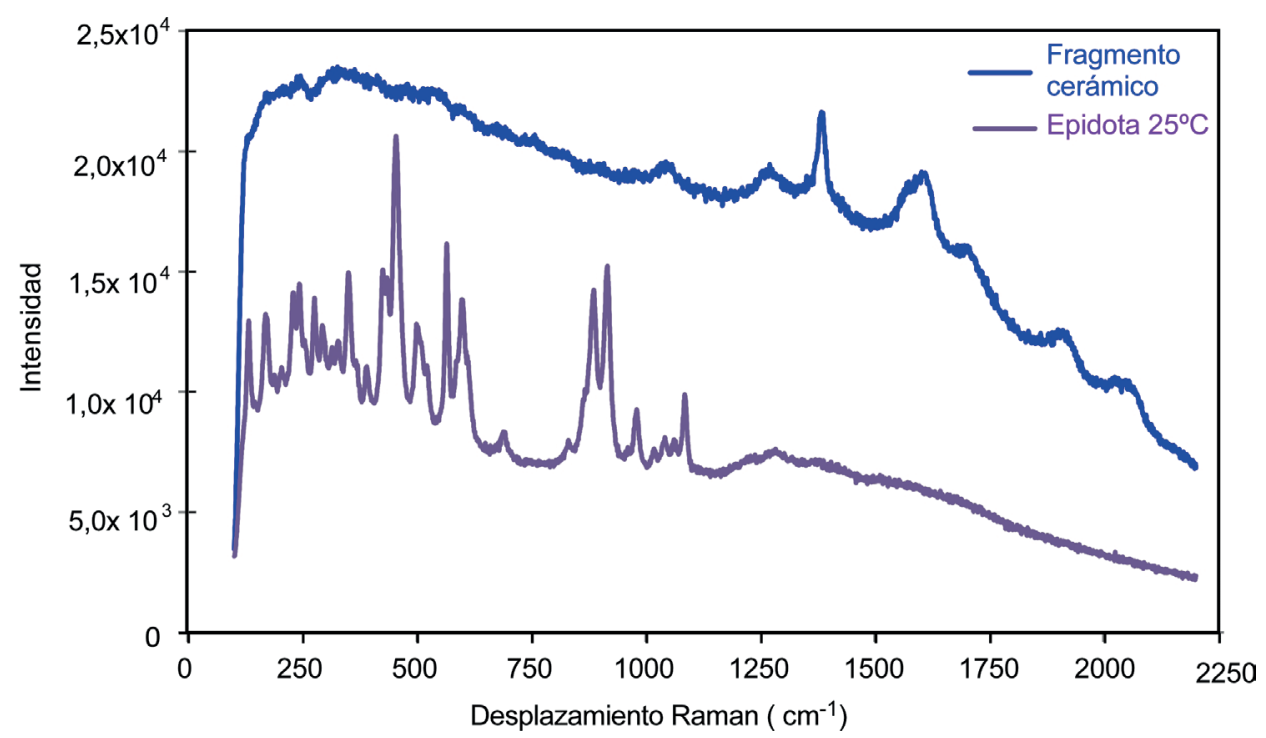

Figura 9. Espectros Raman medidos sobre la muestra de mineral de epidota recolectado en Cerro de Oro y el polvo raspado de la superficie de uno de los fragmentos cerámicos. Los espectros se tomaron usando un láser de $785 \mathrm{~nm}$.

\subsection{Termoalteración a altas temperaturas del mineral verdoso}

Difracción de rayos X (DRX)

Existen evidencias de cambios estructurales en la epidota cuando esta se calienta por encima de $900{ }^{\circ} \mathrm{C}$. Según la bibliografía (Gatta et al. 2011), se espera que, por encima de esa temperatura, 


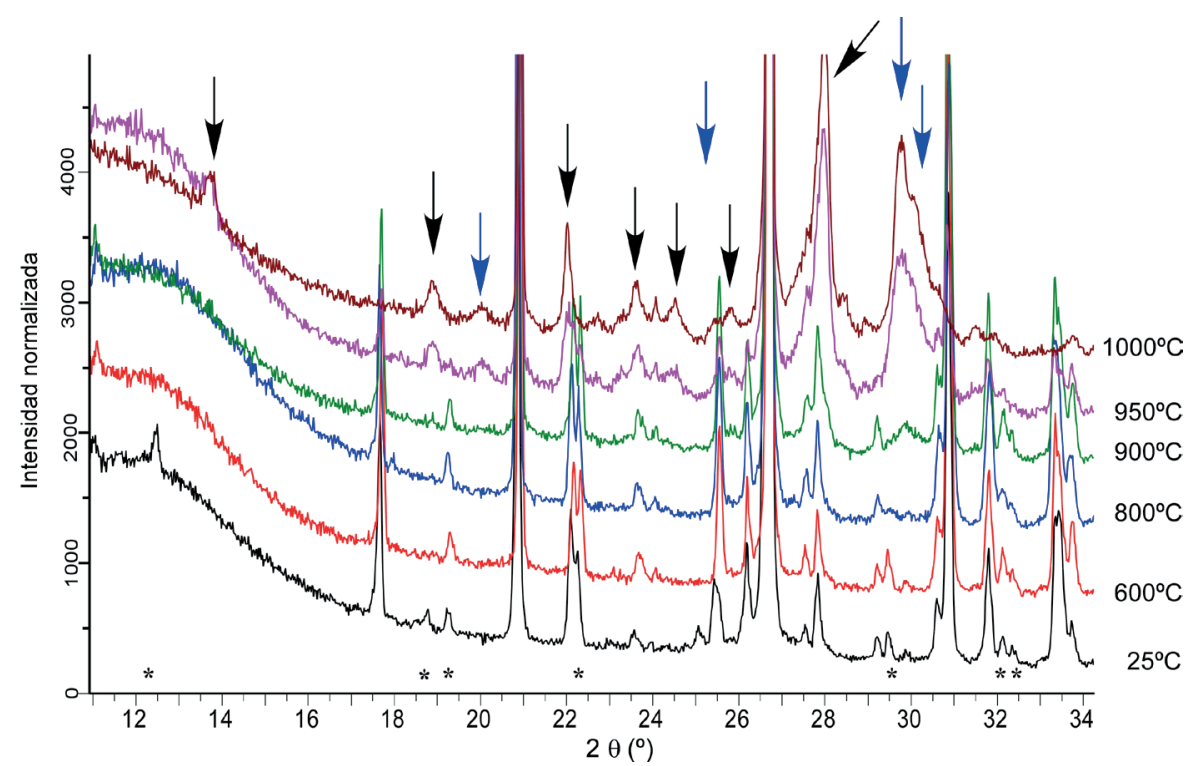

Figura 10. Difractogramas en polvo de las muestras de roca molidas y calentadas a las temperaturas indicadas en cada uno de los diagramas. Los asteriscos indican los máximos de difracción de la epidota que desaparecen mientras que las flechas azules muestran los máximos correspondientes a un diópsido y las flechas negras son las correspondientes a la anortita.

la epidota pura se descomponga a una mezcla de anortita y un piroxeno con la posible formación de $\mathrm{Al}_{2} \mathrm{O}_{3}, \mathrm{Fe}_{2} \mathrm{O}_{3}$ y pérdida de agua. Por esta razón se realizaron análisis de difracción de rayos $\mathrm{X}$ buscando observar cambios en la estructura y composición de la epidota una vez que esta fue alterada a las temperaturas indicadas previamente. La figura 10 muestra los cambios en los difractogramas obtenidos después de moler las pastillas formadas con los fragmentos de roca molidos después de haber sido calentadas a las temperaturas indicadas. Como puede observarse, la descomposición de la epidota comienza por encima de $900{ }^{\circ} \mathrm{C}$ y, a $1000{ }^{\circ} \mathrm{C}$, se observa la presencia de diópsido (PDF-75-1092) y anortita (PDF-41-1486), tal como se esperaba.

\section{Microscopía Raman}

Una vez que se confirmó que la epidota se descompone a altas temperaturas y que la presencia de cuarzo no altera los productos de descomposición ya reportados, se procedió a obtener espectros Raman a ciertas temperaturas seleccionadas: antes y después de las transformaciones estructurales $\left(600{ }^{\circ} \mathrm{C}, 800{ }^{\circ} \mathrm{C}\right.$ y $\left.1000{ }^{\circ} \mathrm{C}\right)$. Los espectros se muestran en la figura 11a. Como puede observarse, los espectros de las pastillas calentadas a $600{ }^{\circ} \mathrm{C}$ y $800{ }^{\circ} \mathrm{C}$, son similares al de la epidota natural sin termoalterar, aunque a $600{ }^{\circ} \mathrm{C}$ aparece un pico extra (aproximadamente a $1390 \mathrm{~cm}^{-1}$ ) y a $800{ }^{\circ} \mathrm{C}$ se observa otro más a $1430 \mathrm{~cm}^{-1}$. Esto nos indica que la epidota podría estar experimentando cambios incluso a temperaturas inferiores a su descomposición química. Se puede observar que la muestra de epidota calentada a $1000{ }^{\circ} \mathrm{C}$ presenta un espectro muy diferente al de las otras muestras, consistente con la descomposición de la epidota observada por DRX. Esto nos indica que, si este pigmento fue usado sobre la pasta cerámica y luego fue cocido, no es posible comparar los espectros Raman del mineral natural sin alterar térmicamente y los del fragmento raspado, la comparación debe hacerse con muestras de mineral termoalterado.

Al comparar los espectros Raman de las epidotas calentadas a diversas temperaturas con los del pigmento raspado de un fragmento (Fig. 11b), se observan ciertas características comunes en el rango de $1200 \mathrm{a} 2200 \mathrm{~cm}^{-1}$. Esto sugiere que el pigmento utilizado en la cerámica puede haber 

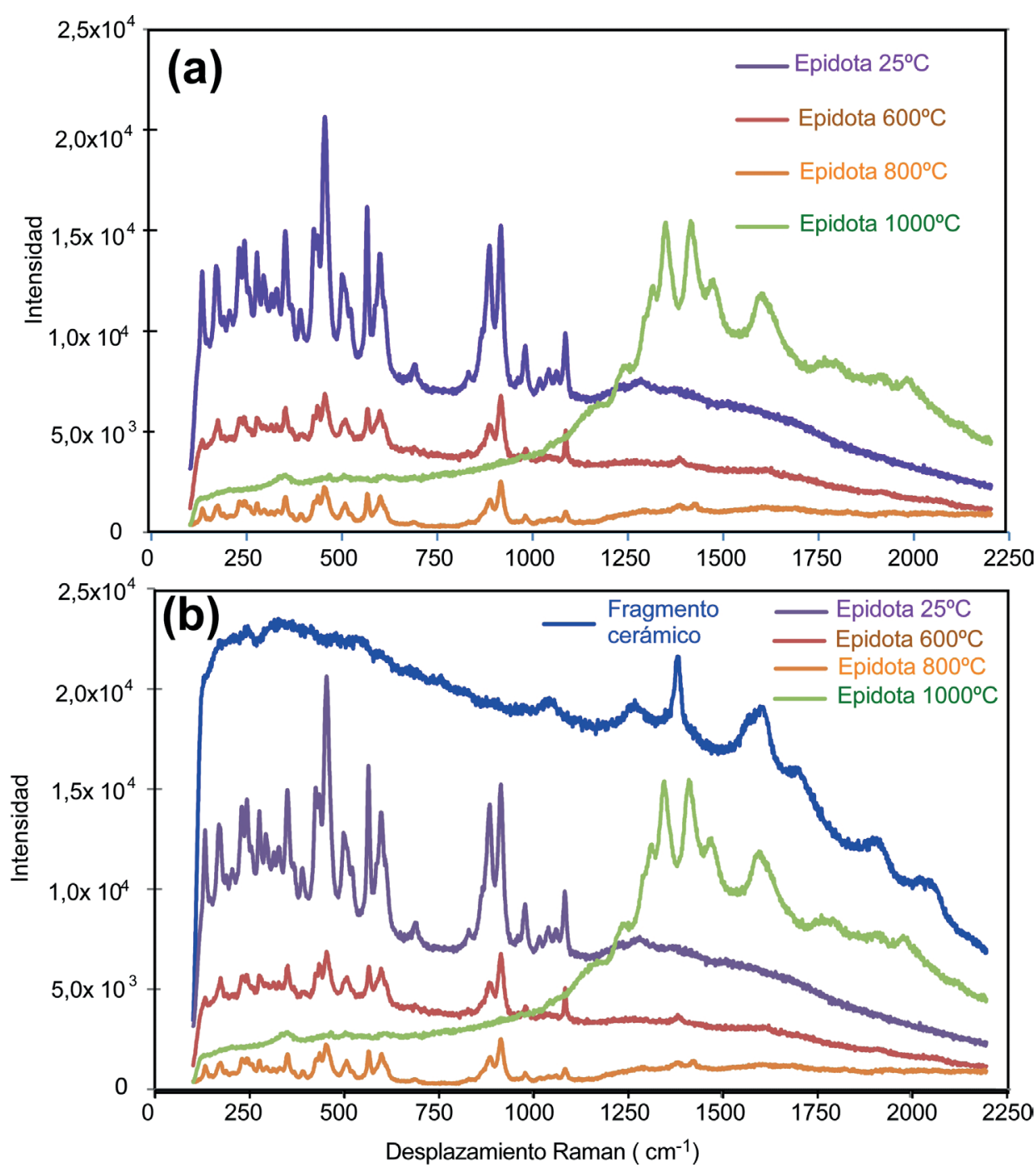

Figura 11. Espectros Raman medidos sobre los granos verdes en las pastillas molidas y calentadas a diferentes temperaturas. Los espectros se tomaron usando un láser de $785 \mathrm{~nm}$.

estado compuesto de epidota. Se hace necesario medir espectros Raman en pastillas calentadas a temperaturas intermedias con el fin de encontrar una posible temperatura en la cual se observe una mayor coincidencia entre los espectros, ya que los cambios estructurales, según la información de difracción de rayos $\mathrm{X}$, no son bruscos, sino que avanzan de manera progresiva según se aumenta la temperatura. El intervalo de temperatura a estudiar comienza desde $600{ }^{\circ} \mathrm{C}$ y se extiende hasta $1000^{\circ} \mathrm{C}$, lo cual cubre el intervalo estimado de temperaturas de cocción en Cerro de Oro (Fernandini et al. 2019).

Además, cabe señalar, que se produjeron cambios en la coloración del pigmento mineral a altas temperaturas, sobre todo en las pastillas de $900{ }^{\circ} \mathrm{C}-1000{ }^{\circ} \mathrm{C}$. Estos cambios en el color del pigmento mineral van del verde pistacho pálido original del mineral sin calentar al verde amarillento en piezas calentadas a altas temperaturas. Esta variación de tonos también está representada en el repertorio cerámico de Cerro de Oro (Fig. 2). Es por ello por lo que se ha decidido no caracterizar este color a partir de estándares como el sistema de color Munsell o un colorímetro. 
La presencia de estos tonos más amarillentos, vinculados con el calentamiento del mineral, podría ser un indicador más del uso potencial de la epidota como el pigmento usado en Cerro de Oro y su repertorio cerámico.

\section{Conclusiones y propuestas a futuro}

La relación entre el pigmento cerámico y la epidota, así como la consistencia del color verde amarillento en el repertorio cerámico de Cerro de Oro nos invita a pensar en el mineral epidota como una de las materias primas más importantes en la paleta cromática del repertorio cerámico del sitio. La evidencia muestra que la epidota tratada a altas temperaturas ofrece espectros Raman compatibles con el espectro de la muestra extraída de los fragmentos cerámicos. La identificación de este pigmento en el repertorio cerámico andino es novedosa y apenas se tienen registros de la utilización de este pigmento (Newman y Derrick 2002). Identificar el origen y uso de este pigmento, nos permite además ahondar en la definición cultural de Cerro de Oro, así como de su importancia en el desarrollo cultural de Cañete durante el Horizonte Medio. La observación de este pigmento en otros sitios arqueológicos cercanos y la presencia de este color en sus repertorios cerámicos, nos invitan a pensar en un fenómeno regional. Cabe destacar que la pieza estudiada ha sido relativa y absolutamente ubicada en una fase de ocupación durante la cual Cerro de Oro se caracteriza por una dinámica relación con sus vecinos, particularmente en términos de influencia en diseños cerámicos. En este sentido, la amplia prevalencia del uso del color verde amarillento en el repertorio cerámico del sitio, aparentemente extraído del mismo sitio, nos permite entender al estilo Cerro de Oro como un proceso mediante el cual se incorporaban elementos foráneos mientras que se mantenía un fuerte arraigo local.

Si bien los resultados de este análisis son prometedores, se requieren estudios más detallados del mineral a altas temperaturas, así como más análisis en torno al pigmento cerámico. Para ello, se llevarán a cabo análisis más detallados del mineral calentado a altas temperaturas. Por un lado, entre $600{ }^{\circ} \mathrm{C}$ y $800{ }^{\circ} \mathrm{C}$ para comprender la aparición del pico Raman de $-1390 \mathrm{~cm}^{-1}$ que coincide con uno de los picos del pigmento verde usado. Y, por otro lado, en la región de los cambios estructurales observados por DRX, especialmente entre $900{ }^{\circ} \mathrm{C}$ y $1000{ }^{\circ} \mathrm{C}$, para poder entender mejor esta región de temperaturas, ya que los espectros Raman del mineral medidos a $1000^{\circ} \mathrm{C}$ también muestran algunos máximos similares con el pigmento cerámico. Además, se llevarán a cabo más análisis del pigmento cerámico con distintas muestras del repertorio cerámico del sitio.

\section{Agradecimientos}

Agradecemos la ayuda prestada por Julio Phan durante los análisis iniciales de esta investigación. También a Gabriela De la Puente y Alexandra Oshiro por su ayuda en la elaboración de figuras del repertorio cerámico. Se agradece también a Martín Fuentes, por su ayuda preliminar en la identificación del mineral. Finalmente, agradecemos al Centro de Caracterización de Materiales (CAMPUCP) de la Pontificia Universidad Católica del Perú por facilitarnos el acceso y uso de los equipos de difracción de rayos $\mathrm{X}$ y de espectroscopía Raman indicados en el texto.

\section{Referencias}

Boletin de Arqueología PUCP (2019). Avances en el análisis de cerámica y pigmentos en arqueología. Parte 1. Boletín de Arqueología PUCP (2019). Avances en el análisis de cerámica y pigmentos en arqueología. Parte 2.

Chapoulie, R., Sepúlveda, M., Del-Solar-Velarde N. y V. Wright (eds.) (2018). Arqueometría: estudios analíticos de materiales arqueológicos, Instituto Francés de Estudios Andinos (IFEA)/Université Bordeaux Montaigne/ Universidad de Tarapacá, Lima. 
Del-Solar-Velarde, N., R. Chapoulie y L.J. Castillo Butters (2019). Análisis químico y mineralógico de pinturas en cerámicas arqueológicas del valle de Jequetepeque y sus implicancias arqueológicas, Boletín de Arqueología PUCP 26, 33-47. https://doi.org/10.18800/boletindearqueologiapucp.201901.002

Dulanto, J., P. Gonzáles y E. Guadalupe (2019). Los pigmentos utilizados en la pintura poscocción de vasijas de cerámica Paracas Temprano de Puerto Nuevo y las redes de intercambio de la costa y sierra surcentral del Perú durante los siglos IX-VI a.C., Boletin de Arqueología PUCP 26, 65-83. https://doi.org/10.18800/boletindearqueologiapucp.201901.004

Fernandini, F. (2015). Beyond the Empire: Living in Cerro de Oro. PhD dissertation. California: Department of Anthropology, Stanford University, Stanford.

Fernandini, F. (2018a). Informe Final del Proyecto de Investigación Arqueológica Cerro de Oro, Temporada 2017. Lima: Ministerio de Cultura.

Fernandini, F. (2018b). Peopling the Cañete valley circa AD 600: A view from Cerro de Oro, Nawpa Pacha 38(2), 135-156. https://doi.org/10.1080/00776297.2018.1512196

Fernandini, F. (2020). Identidades heterogéneas: construyendo una persona funeraria en Cerro de Oro. Artículo en preparación, en: R. Vega Centeno y J. Dulanto (eds.), Los desafios del tiempo, el espacio y la memoria. Ensayos en homenaje a Peter Kaulicke, Fondo Editorial PUCP, Lima.

Fernandini, F., A. Trujillo Quinde, M. Mejía Santillán y S. Cuadros Gómez (2019). Caracterizando la cerámica de Cerro de Oro por espectroscopía Mössbauer y difracción de rayos-x, Boletín de Arqueología PUCP 27, 49-66. https://doi.org/10.18800/boletindearqueologiapucp.201902.003

Gatta, G.D., M. Merlini, Y. Lee y S. Poli (2011). Behavior of epidote at high pressure and high temperature: A powder diffraction study up to $10 \mathrm{GPa}$ and 1,200 K, Physics and Chemistry of Minerals 38, 419-428. https:// doi.org/10.1007/s00269-010-0415-y

González-Gómez de Agüero, A. (2019). Compartiendo mesa e identidades: un estudio de dieta y comensalidad en Cerro de Oro, tesis de maestría, Escuela de Posgrado, Pontificia Universidad Católica del Perú, Lima.

Klein, C. y C.S. Hurlbut (1998). Manual de mineralogía, tomo 1 y 2, Reverté, Barcelona.

Kremer (s/f). https://www.kremer-pigmente.com/es/pigmentos/pigmentos-propios-e-historicos/103/epidotapiedra

Kriss, D., E. Howe, J. Levinson, A. Rizzo, F. Carò y L. DeLeonardis (2018). A material and technical study of Paracas painted ceramics, Antiquity 92(366), 1492-1510. https://doi.org/10.15184/aqy.2018.164

Kroeber, A. (1937). Archaeological Explorations in Peru. Part IV. Cañete Valley, Fieldiana, Anthropology Memoirs 2(4), Field Museum of Natural History, Chicago.

Lafuente, B., R.T. Downs, H. Yang y N. Stone (2015). The power of databases: the RRUFF project, en: T. Armbruster y R.M. Danisi (eds.), Highlights in Mineralogical Crystallography, 1-30, De Gruyter, Berlin. https:// doi.org/rruff-2.geo.arizona.edu/epidote/R050191

Menzel, D. (1964). Style and time in the Middle Horizon, Nawpa Pacha 1, 1-105.

Newman, R. y M. Derrick (2002). Painted Qero cups from the Inka and Colonial periods in Peru: An analytical study of pigments and media, MRS Proceedings 712, II9.8. https://doi.org/10.1557/PROC-712-II9.8

Rodríguez, C. (2017). Los objetos como cosas: una aproximación arqueológica desde Cerro de Oro, tesis de licenciatura, Facultad de Letras y Ciencias Humanas, Pontificia Universidad Católica del Perú, Lima.

Ruales, M. (2000). Investigaciones en Cerro del Oro, Valle de Cañete, Boletín de Arqueología PUCP 4, 359-399.

Salazar, H. y C. Landa (1993). Geología de los cuadrángulos de Mala, Lunahuaná, Tupe, Conayca, Chincha, Tantará y Castrovirreyna 26-j, 26-k, 26-1, 26-m, 27-k, 27-1, 27-m - [Boletín A 44]. Serie A: Carta geológica nacional. República del Perú. Sector Energía y Minas. Instituto Geológico Minero y Metalúrgico. Lima, Perú.

Sepúlveda, M y E. Casanova (eds.) (2020). Pigments, dyes, and colors in Latin American archaeometric investigations, Heritage Science. Recuperado de https://www.springeropen.com/collections/PDCLAAI

Stumer, L. (1971). Informe preliminar sobre el recorrido del Valle de Cañete, Arqueología y Sociedad, 5, 23-35.

Velde, B. e I.C. Druc (1999). Archaeological Ceramic Materials. Origin and Utilization, Springer-Verlag, Berlín

Wright, V. (2010). Pigmentos y tecnología artística mochicas: una nueva aproximación en la comprensión de la organización social, Bulletin de l'Institut Français d'Études Andines 39(2), 299-330.

Recibido: 21/07/2020

Aprobado: 12/10/2020 